\title{
Some Common Fixed Point Theorems in Complex Valued $b$-Metric Spaces
}

\author{
Aiman A. Mukheimer \\ Department of Mathematical Sciences, Prince Sultan University, Riyadh 11586, Saudi Arabia \\ Correspondence should be addressed to Aiman A. Mukheimer; mukheimer@oyp.psu.edu.sa
}

Received 6 January 2014; Accepted 12 March 2014; Published 25 May 2014

Academic Editors: A. Alotaibi and S. A. Mohiuddine

Copyright (C) 2014 Aiman A. Mukheimer. This is an open access article distributed under the Creative Commons Attribution License, which permits unrestricted use, distribution, and reproduction in any medium, provided the original work is properly cited.

Azam et al. (2011), introduce the notion of complex valued metric spaces and obtained common fixed point result for mappings in the context of complex valued metric spaces. Rao et al. (2013) introduce the notion of complex valued $b$-metric spaces. In this paper, we generalize the results of Azam et al. (2011), and Bhatt et al. (2011), by improving the conditions of contraction to establish the existence and uniqueness of common fixed point for two self-mappings on complex valued $b$-metric spaces. Some examples are given to illustrate the main results.

\section{Introduction}

Banach contraction principle in [1] was the starting point for many researchers during last decades in the field of nonlinear analysis. In 1989, Bakhtin [2] introduced the concept of $b$ metric space as a generalization of metric spaces. The concept of complex valued $b$-metric spaces was introduced in 2013 by Rao et al. [3], which was more general than the well-known complex valued metric spaces that were introduced in 2011 by Azam et al. [4]. The main purpose of this paper is to present common fixed point results of two self-mappings satisfying a rational inequality on complex valued $b$-metric spaces. The results presented in this paper are generalization of work done by Azam et al. in [4] and Bhatt et al in [5].

Definition 1 (see [6]). Let $X$ be a nonempty set and let $s \geq 1$ be a given real number. A function $d: X \times X \rightarrow[0, \infty)$ is called a $b$-metric if for all $x, y, z \in X$ the following conditions are satisfied:

(i) $d(x, y)=0$ if and only if $x=y$;

(ii) $d(x, y)=d(y, x)$;

(iii) $d(x, y) \leq s[d(x, z)+d(z, y)]$.

The pair $(X, d)$ is called a $b$-metric space. The number $s \geq 1$ is called the coefficient of $(X, d)$.
Example 2 (see [7]). Let $(X, d)$ be a metric space and $\rho(x, y)=(d(x, y))^{p}$, where $p>1$ is a real number. Then $(X, \rho)$ is a $b$-metric space with $s=2^{p-1}$.

Let $\mathbb{C}$ be the set of complex numbers and $z_{1}, z_{2} \in \mathbb{C}$. Define a partial order $\lesssim$ on $\mathbb{C}$ as follows:

$$
\begin{gathered}
z_{1} \precsim z_{2} \\
\text { iff } \operatorname{Re}\left(z_{1}\right) \leq \operatorname{Re}\left(z_{1}\right), \quad \operatorname{Im}\left(z_{1}\right) \leq \operatorname{Im}\left(z_{2}\right) .
\end{gathered}
$$

Thus $z_{1} \precsim z_{2}$ if one of the following holds:

(1) $\operatorname{Re}\left(z_{1}\right)=\operatorname{Re}\left(z_{2}\right)$ and $\operatorname{Im}\left(z_{1}\right)=\operatorname{Im}\left(z_{2}\right)$,

(2) $\operatorname{Re}\left(z_{1}\right)<\operatorname{Re}\left(z_{2}\right)$ and $\operatorname{Im}\left(z_{1}\right)=\operatorname{Im}\left(z_{2}\right)$,

(3) $\operatorname{Re}\left(z_{1}\right)=\operatorname{Re}\left(z_{2}\right)$ and $\operatorname{Im}\left(z_{1}\right)<\operatorname{Im}\left(z_{2}\right)$,

(4) $\operatorname{Re}\left(z_{1}\right)<\operatorname{Re}\left(z_{2}\right)$ and $\operatorname{Im}\left(z_{1}\right)<\operatorname{Im}\left(z_{2}\right)$

We will write $z_{1} \precsim z_{2}$ if $z_{1} \neq z_{2}$ and one of (2), (3), and (4) is satisfied; also we will write $z_{1} \prec z_{2}$ if only (4) is satisfied.

Remark 3. We can easily check that the following statements are held:

(i) if $a, b \in \mathbb{R}$ and $a \leq b$, then $a z \lesssim b z$ for all $z \in \mathbb{C}$;

(ii) if $0 \precsim z_{1} \precsim z_{2}$, then $\left|z_{1}\right|<\left|z_{2}\right|$; 
(iii) if $z_{1} \precsim z_{2}$ and $z_{2} \prec z_{3}$, then $z_{1} \prec z_{3}$.

Definition 4 (see [4]). Let $X$ be a nonempty set. A function $d: X \times X \rightarrow \mathbb{C}$ is called a complex valued metric on $X$ if for all $x, y, z \in X$ the following conditions are satisfied:

(i) $0 \precsim d(x, y)$ and $d(x, y)=0$ if and only if $x=y$;

(ii) $d(x, y)=d(y, x)$;

(iii) $d(x, y) \lesssim d(x, z)+d(z, y)$.

The pair $(X, d)$ is called a complex valued metric space.

Example 5 (see [8]). Let $X=\mathbb{C}$. Define the mapping $d: X \times$ $X \rightarrow \mathbb{C}$ by

$$
d(x, y)=i|x-y|, \quad \forall x, y \in X
$$

Then $(X, d)$ is a complex valued metric space.

Example 6 (see [9]). Let $X=\mathbb{C}$. Define the mapping $d: X \times$ $X \rightarrow \mathbb{C}$ by

$$
d(x, y)=e^{i k}|x-y|, \quad \text { where } k \in \mathbb{R}, \forall x, y \in X .
$$

Then $(X, d)$ is a complex valued metric space.

Definition 7 (see [3]). Let $X$ be a nonempty set and let $s \geq$ 1 be a given real number. A function $d: X \times X \rightarrow \mathbb{C}$ is called a complex valued $b$-metric on $X$ if for all $x, y, z \in X$ the following conditions are satisfied:

(i) $0 \precsim d(x, y)$ and $d(x, y)=0$ if and only if $x=y$;

(ii) $d(x, y)=d(y, x)$;

(iii) $d(x, y) \lesssim s[d(x, z)+d(z, y)]$.

The pair $(X, d)$ is called a complex valued $b$-metric space.

Example 8 (see [3]). Let $X=[0,1]$. Define the mapping $d$ : $X \times X \rightarrow \mathbb{C}$ by

$$
d(x, y)=|x-y|^{2}+i|x-y|^{2}, \quad \forall x, y \in X .
$$

Then $(X, d)$ is a complex valued $b$-metric space with $s=2$.

Definition 9 (see [3]). Let $(X, d)$ be a complex valued $b$-metric space. Consider the following.

(i) A point $x \in X$ is called interior point of a set $A \subseteq X$ whenever there exists $0 \prec r \in \mathbb{C}$ such that $B(x, r):=$ $\{y \in X: d(x, y) \prec r\} \subseteq A$.

(ii) A point $x \in X$ is called a limit point of a set $A$ whenever, for every $0 \prec r \in \mathbb{C}, B(x, r) \cap(A-X) \neq \emptyset$.

(iii) A subset $A \subseteq X$ is called open whenever each element of $A$ is an interior point of $A$.

(iv) A subset $A \subseteq X$ is called closed whenever each element of $A$ belongs to $A$.

(v) A subbasis for a Hausdorff topology $\tau$ on $X$ is a family $F=\{B(x, r): x \in X$ and $0 \prec r\}$.
Definition 10 (see [3]). Let $(X, d)$ be a complex valued $b$ metric space and $\left\{x_{n}\right\}$ a sequence in $X$ and $x \in X$. Consider the following.

(i) If for every $c \in \mathbb{C}$, with $0 \prec r$, there is $N \in \mathbb{N}$ such that, for all $n>\mathbb{N}, d\left(x_{n}, x\right) \prec c$, then $\left\{x_{n}\right\}$ is said to be convergent, $\left\{x_{n}\right\}$ converges to $x$, and $x$ is the limit point of $\left\{x_{n}\right\}$. We denote this by $\lim _{n \rightarrow \infty} x_{n}=x$ or $\left\{x_{n}\right\} \rightarrow x$ as $n \rightarrow \infty$.

(ii) If for every $c \in \mathbb{C}$, with $0 \prec r$, there is $N \in \mathbb{N}$ such that, for all $n>\mathbb{N}, d\left(x_{n}, x_{n+m}\right) \prec c$, where $m \in \mathbb{N}$, then $\left\{x_{n}\right\}$ is said to be Cauchy sequence.

(iii) If every Cauchy sequence in $X$ is convergent, then $(X, d)$ is said to be a complete complex valued $b$ metric space.

Lemma 11 (see [3]). Let $(X, d)$ be a complex valued b-metric space and let $\left\{x_{n}\right\}$ be a sequence in $X$. Then $\left\{x_{n}\right\}$ converges to $x$ if and only if $\left|d\left(x_{n}, x\right)\right| \rightarrow 0$ as $n \rightarrow \infty$.

Lemma 12 (see [3]). Let $(X, d)$ be a complex valued b-metric space and let $\left\{x_{n}\right\}$ be a sequence in $X$. Then $\left\{x_{n}\right\}$ is a Cauchy sequence if and only if $\left|d\left(x_{n}, x_{n+m}\right)\right| \rightarrow 0$ as $n \rightarrow \infty$, where $m \in \mathbb{N}$.

Theorem 13 (see [4]). Let $(X, d)$ be a complete complex valued metric space and let $\lambda, \mu$ be nonnegative real numbers such that $\lambda+\mu<1$. Suppose that $S, T: X \rightarrow X$ are mappings satisfying

$$
d(S x, T y) \lesssim \lambda d(x, y)+\frac{\mu d(x, S x) d(y, T y)}{1+d(x, y)},
$$

for all $x, y \in X$. Then $S, T$ have a unique common fixed point in $X$.

Theorem 14 (see [5]). Let $(X, d)$ be a complete complex valued metric space and let $S, T: X \rightarrow X$ be mappings satisfying

$$
d(S x, T y) \precsim \frac{a[d(x, S x) d(x, T y)+d(y, T y) d(y, S x)]}{d(x, T y)+d(y, S x)},
$$

for all $x, y \in X$, where $a \in[0,1)$. Then $S, T$ have a unique common fixed point in $X$.

\section{Main Result}

Our next theorem is a generalization of Theorem 13 in complex valued $b$-metric spaces.

Theorem 15. Let $(X, d)$ be a complete complex valued b-metric space with the coefficient $s \geq 1$ and let $S, T: X \rightarrow X$ be mappings satisfying

$$
d(S x, T y) \precsim \lambda d(x, y)+\frac{\mu d(x, S x) d(y, T y)}{1+d(x, y)},
$$

for all $x, y \in X$, where $\lambda, \mu$ are nonnegative reals with $s \lambda+\mu<$ 1. Then $S, T$ have a unique common fixed point in $X$. 
Proof. For any arbitrary point, $x_{0} \in X$. Define sequence $\left\{x_{n}\right\}$ in $X$ such that

$$
x_{2 n+1}=S x_{2 n}, x_{2 n+2}=T x_{2 n+1}, \quad \text { for } n=0,1,2,3, \ldots .
$$

Now, we show that the sequence $\left\{x_{n}\right\}$ is Cauchy. Let $x=x_{2 n}$ and $y=x_{2 n+1}$ in (7); we have

$$
\begin{aligned}
& d\left(x_{2 n+1}, x_{2 n+2}\right) \\
& \quad=d\left(S x_{2 n}, T x_{2 n+1}\right) \\
& \quad \lesssim \lambda d\left(x_{2 n}, x_{2 n+1}\right)+\frac{\mu d\left(x_{2 n}, S x_{2 n}\right) d\left(x_{2 n+1}, T x_{2 n+1}\right)}{1+d\left(x_{2 n}, x_{2 n+1}\right)} \\
& =\lambda d\left(x_{2 n}, x_{2 n+1}\right)+\frac{\mu d\left(x_{2 n}, x_{2 n+1}\right) d\left(x_{2 n+1}, x_{2 n+2}\right)}{1+d\left(x_{2 n}, x_{2 n+1}\right)},
\end{aligned}
$$

which implies that

$$
\begin{aligned}
& \left|d\left(x_{2 n+1}, x_{2 n+2}\right)\right| \\
& \quad \leq \lambda\left|d\left(x_{2 n}, x_{2 n+1}\right)\right|+\frac{\mu\left|d\left(x_{2 n}, x_{2 n+1}\right)\right|\left|d\left(x_{2 n+1}, x_{2 n+2}\right)\right|}{\left|1+d\left(x_{2 n}, x_{2 n+1}\right)\right|} .
\end{aligned}
$$

Since $\left|1+d\left(x_{2 n}, x_{2 n+1}\right)\right|>\left|d\left(x_{2 n}, x_{2 n+1}\right)\right|$, we get

$$
\begin{aligned}
& \left|d\left(x_{2 n+1}, x_{2 n+2}\right)\right| \\
& \quad \leq \lambda\left|d\left(x_{2 n}, x_{2 n+1}\right)\right|+\mu\left|d\left(x_{2 n+1}, x_{2 n+2}\right)\right|,
\end{aligned}
$$

and hence

$$
\left|d\left(x_{2 n+1}, x_{2 n+2}\right)\right| \leq \frac{\lambda}{1-\mu}\left|d\left(x_{2 n}, x_{2 n+1}\right)\right| .
$$

Similarly, we obtain

$$
\left|d\left(x_{2 n+2}, x_{2 n+3}\right)\right| \leq \frac{\lambda}{1-\mu}\left|d\left(x_{2 n+1}, x_{2 n+2}\right)\right| .
$$

Since $s \lambda+\mu<1$ and $s \geq 1$, we get $\lambda+\mu<1$.

Therefore, with $\delta=\lambda /(1-\mu)<1$, and for all $n \geq 0$, consequently, we have

$$
\begin{aligned}
& \left|d\left(x_{2 n+1}, x_{2 n+2}\right)\right| \\
& \quad \leq \delta\left|d\left(x_{2 n}, x_{2 n+1}\right)\right| \leq \delta^{2}\left|d\left(x_{2 n-1}, x_{2 n}\right)\right| \\
& \quad \leq \cdots \leq \delta^{2 n+1}\left|d\left(x_{0}, x_{1}\right)\right| .
\end{aligned}
$$

Thus for any $m>n, m, n \in \mathbb{N}$, and since $s \delta=s \lambda /(1-\mu)<1$, we get

$$
\begin{aligned}
& \left|d\left(x_{n}, x_{m}\right)\right| \\
& \leq s\left|d\left(x_{n}, x_{n+1}\right)\right|+s\left|d\left(x_{n+1}, x_{m}\right)\right| \\
& \leq s\left|d\left(x_{n}, x_{n+1}\right)\right|+s^{2}\left|d\left(x_{n+1}, x_{n+2}\right)\right|+s^{2}\left|d\left(x_{n+2}, x_{m}\right)\right| \\
& \leq s\left|d\left(x_{n}, x_{n+1}\right)\right|+s^{2}\left|d\left(x_{n+1}, x_{n+2}\right)\right|+s^{3}\left|d\left(x_{n+2}, x_{m}\right)\right| \\
& \quad+s^{3}\left|d\left(x_{n+2}, x_{m}\right)\right|
\end{aligned}
$$

$$
\begin{aligned}
\leq & s\left|d\left(x_{n}, x_{n+1}\right)\right|+s^{2}\left|d\left(x_{n+1}, x_{n+2}\right)\right|+s^{3}\left|d\left(x_{n+2}, x_{m}\right)\right| \\
& +\cdots+s^{m-n-2}\left|d\left(x_{m-3}, x_{m-2}\right)\right| \\
& +s^{m-n-1}\left|d\left(x_{m-2}, x_{m-1}\right)\right|+s^{m-n-1}\left|d\left(x_{m-1}, x_{m}\right)\right| \\
\leq & s\left|d\left(x_{n}, x_{n+1}\right)\right|+s^{2}\left|d\left(x_{n+1}, x_{n+2}\right)\right| \\
& +s^{3}\left|d\left(x_{n+2}, x_{m}\right)\right| \\
& +\cdots+s^{m-n-2}\left|d\left(x_{m-3}, x_{m-2}\right)\right| \\
& +s^{m-n-1}\left|d\left(x_{m-2}, x_{m-1}\right)\right|+s^{m-n}\left|d\left(x_{m-1}, x_{m}\right)\right| .
\end{aligned}
$$

By using (14) we get

$$
\begin{aligned}
&\left|d\left(x_{n}, x_{m}\right)\right| \\
& \leq s \delta^{n}\left|d\left(x_{0}, x_{1}\right)\right|+s^{2} \delta^{n+1}\left|d\left(x_{0}, x_{1}\right)\right| \\
&+s^{3} \delta^{n+2}\left|d\left(x_{0}, x_{1}\right)\right| \\
&+\cdots+s^{m-n-2} \delta^{m-3}\left|d\left(x_{0}, x_{1}\right)\right| \\
&+s^{m-n-1} \delta^{m-2}\left|d\left(x_{0}, x_{1}\right)\right|+s^{m-n} \delta^{m-1}\left|d\left(x_{0}, x_{1}\right)\right| \\
&= \sum_{i=1}^{m-n} s^{i} \delta^{i+n-1}\left|d\left(x_{0}, x_{1}\right)\right| .
\end{aligned}
$$

Therefore,

$$
\begin{aligned}
& \left|d\left(x_{n}, x_{m}\right)\right| \\
& \quad \leq \sum_{i=1}^{m-n} s^{i+n-1} \delta^{i+n-1}\left|d\left(x_{0}, x_{1}\right)\right| \\
& \quad=\sum_{t=n}^{m-1} s^{t} \delta^{t}\left|d\left(x_{0}, x_{1}\right)\right| \\
& \leq \sum_{t=n}^{\infty}(s \delta)^{t}\left|d\left(x_{0}, x_{1}\right)\right| \\
& \quad=\frac{(s \delta)^{n}}{1-s \delta}\left|d\left(x_{0}, x_{1}\right)\right|,
\end{aligned}
$$

and hence

$$
\left|d\left(x_{n}, x_{m}\right)\right| \leq \frac{(s \delta)^{n}}{1-s \delta}\left|d\left(x_{0}, x_{1}\right)\right| \longrightarrow 0 \quad \text { as } m, n \longrightarrow \infty .
$$

Thus, $\left\{x_{n}\right\}$ is a Cauchy sequence in $X$.

Since $X$ is complete, there exists some $u \in X$ such that $x_{n} \rightarrow u$ as $n \rightarrow \infty$. Assuming not, then there exist $z \in X$ such that

$$
|d(u, S u)|=|z|>0 \text {. }
$$

So by using the triangular inequality and (7), we get

$$
\begin{aligned}
z= & d(u, S u) \\
\precsim & s d\left(u, x_{2 n+2}\right)+s d\left(x_{2 n+2}, S u\right) \\
= & s d\left(u, x_{2 n+2}\right)+s d\left(T x_{2 n+1}, S u\right) \\
\lesssim & s d\left(u, x_{2 n+2}\right)+s \lambda d\left(u, x_{2 n+2}\right) \\
& +\frac{s \mu d(u, S u) d\left(x_{2 n+1}, T x_{2 n+1}\right)}{1+d\left(u, x_{2 n+2}\right)}
\end{aligned}
$$




$$
\begin{gathered}
=s d\left(u, x_{2 n+2}\right)+s \lambda d\left(u, x_{2 n+2}\right) \\
+\frac{s \mu d(u, S u) d\left(x_{2 n+1}, x_{2 n+2}\right)}{1+d\left(u, x_{2 n+2}\right)}
\end{gathered}
$$

which implies that

$$
\begin{aligned}
|z| & =|d(u, S u)| \\
& \leq s\left|d\left(u, x_{2 n+2}\right)\right|+\frac{s \mu|d(u, S u)|\left|d\left(x_{2 n+1}, x_{2 n+2}\right)\right|}{\left|1+d\left(u, x_{2 n+2}\right)\right|} .
\end{aligned}
$$

Taking the limit of (21) as $n \rightarrow \infty$, we obtain that $|z|=$ $|d(u, S u)| \leq 0$, a contradiction with (19). So $|z|=0$. Hence $S u=u$. Similarly, we obtain $T u=u$.

Now we show that $S$ and $T$ have unique common fixed point of $S$ and $T$. To show this, assume that $u^{*}$ is another common fixed point of $S$ and $T$. Then

$$
\begin{aligned}
d\left(u, u^{*}\right) & =d\left(S u, T u^{*}\right) \\
& \lesssim \lambda d\left(u, u^{*}\right)+\frac{\mu d(u, S u) d\left(u^{*}, T u^{*}\right)}{1+d\left(u, u^{*}\right)} \\
& \prec d\left(u, u^{*}\right) .
\end{aligned}
$$

This implies that $\left|d\left(x, x^{*}\right)\right|<\left|d\left(u, u^{*}\right)\right|$, a contradiction. So $u=u^{*}$ which proves the uniqueness of common fixed point in $X$. This completes the proof.

Corollary 16. Let $(X, d)$ be a complete complex valued $b$ metric space with the coefficient $s \geq 1$ and let $T: X \rightarrow X$ be a mapping satisfying

$$
d(T x, T y) \lesssim \lambda d(x, y)+\frac{\mu d(x, T x) d(y, T y)}{1+d(x, y)},
$$

for all $x, y \in X$, where $\lambda, \mu$ are nonnegative reals with $s \lambda+\mu<$ 1. Then $T$ has a unique fixed point in $X$.

Proof. We can prove this result by applying Theorem 15 with $S=T$.

Corollary 17. Let $(X, d)$ be a complete complex valued $b$ metric space with the coefficient $s \geq 1$ and let $T: X \rightarrow X$ be a mapping satisfying

$$
d\left(T^{n} x, T^{n} y\right) \lesssim \lambda d(x, y)+\frac{\mu d\left(x, T^{n} x\right) d\left(y, T^{n} y\right)}{1+d(x, y)},
$$

for all $x, y \in X$, where $\lambda, \mu$ are nonnegative reals with $s \lambda+\mu<$ 1. Then $T$ has a unique fixed point in $X$.

Proof. From Corollary 20, we obtain $u \in X$ such that

$$
T^{n} u=u
$$

The uniqueness follows from

$$
\begin{aligned}
d(T u, u) & =d\left(T T^{n} u, T^{n} u\right)=d\left(T^{n} T u, T^{n} u\right) \\
& \lesssim \lambda d(T u, u)+\frac{\mu d\left(T u, T^{n} T u\right) d\left(u, T^{n} u\right)}{1+d(T u, u)} \\
& \lesssim \lambda d(T u, u)+\frac{\mu d\left(T u, T^{n} T u\right) d(u, u)}{1+d(T u, u)} \\
& =\lambda d(T u, u) .
\end{aligned}
$$

By taking modulus of (26) and since $\lambda<1$, we obtain $|d(T u, u)| \leq \lambda|d(T u, u)|<|d(T u, u)|$, a contradiction. So, $T u=u$. Hence

$$
T u=T^{n} u=u \text {. }
$$

Therefore, the fixed point of $T$ is unique. This completes the proof.

Example 18. Let $X=\mathbb{C}$. Define a function $d: X \times X \rightarrow \mathbb{C}$ such that

$$
d\left(z_{1}, z_{2}\right)=\left|x_{1}-x_{2}\right|^{2}+i\left|y_{1}-y_{2}\right|^{2},
$$

where $z_{1}=x_{1}+i y_{1}$ and $z_{2}=x_{2}+i y_{2}$.

To verify that $(X, d)$ is a complete complex valued $b$ metric space with $s=2$, it is enough to verify the triangular inequality condition.

Let $z_{1}, z_{2}$, and $z_{3} \in X$; then,

$$
\begin{aligned}
d\left(z_{1}, z_{2}\right) & \\
= & \left|x_{1}-x_{2}\right|^{2}+i\left|y_{1}-y_{2}\right|^{2} \\
= & \left|x_{1}-x_{3}+x_{3}-x_{2}\right|^{2}+i\left|y_{1}-y_{3}+y_{3}-y_{2}\right|^{2} \\
\lesssim & \left|x_{1}-x_{3}\right|^{2}+\left|x_{3}-x_{2}\right|^{2}+2\left|x_{1}-x_{3}\right|\left|x_{3}-x_{2}\right| \\
& +i\left[\left|y_{1}-y_{3}\right|^{2}+\left|y_{3}-y_{2}\right|^{2}+2\left|y_{1}-y_{3}\right|\left|y_{3}-y_{2}\right|\right] \\
\precsim & \left|x_{1}-x_{3}\right|^{2}+\left|x_{3}-x_{2}\right|^{2}+\left|x_{1}-x_{3}\right|^{2}+\left|x_{3}-x_{2}\right|^{2} \\
& +i\left[\left|y_{1}-y_{3}\right|^{2}+\left|y_{3}-y_{2}\right|^{2}+\left|y_{1}-y_{3}\right|^{2}+\left|y_{3}-y_{2}\right|^{2}\right] \\
= & 2\left\{\left|x_{1}-x_{3}\right|^{2}+\left|x_{3}-x_{2}\right|^{2}+i\left[\left|y_{1}-y_{3}\right|^{2}+\left|y_{3}-y_{2}\right|^{2}\right]\right\} \\
= & 2\left[d\left(z_{1}, z_{3}\right)+d\left(z_{3}, z_{2}\right)\right] .
\end{aligned}
$$

Therefore, $s=2$.

Now, define two self-mappings $S, T: X \rightarrow X$ as follows:

$$
T z=T(x+i y)= \begin{cases}0 & \text { if } x, y \in \mathbb{Q} \\ 2 & \text { if } x \in \mathbb{Q}^{c}, y \in \mathbb{Q} \\ 2 i & \text { if } x \in \mathbb{Q}^{c}, y \in \mathbb{Q}^{c} \\ 2+2 i & \text { if } x \in \mathbb{Q}, y \in \mathbb{Q}^{c}\end{cases}
$$

such that $S=T$ and $z=x+i y$. Let $x=1 / \pi$ and $y=0$, and since $\lambda \in[0,1)$, we have

$$
\begin{aligned}
d(T x, T y) & =d\left(T \frac{1}{\pi}, T 0\right) \\
& =d(2,0)=4>\lambda \frac{1}{\pi^{2}} \\
& =\lambda d\left(\frac{1}{\pi}, 0\right)+\frac{\mu d(1 / \pi, T(1 / \pi)) d(0, T 0)}{1+d(1 / \pi, 0)} .
\end{aligned}
$$


Note that $T^{n} z=0$ for $n>1$, so

$$
d\left(T^{n} x, T^{n} y\right)=0 \precsim \lambda d(x, y)+\frac{\mu d\left(x, T^{n} x\right) d\left(y, T^{n} y\right)}{1+d(x, y)},
$$

for all $x, y \in X$ and $\lambda, \mu \geq 0$ with $2 \lambda+\mu<1$. So all conditions of Corollary 21 are satisfied to get a unique fixed point 0 of $T$.

Our next theorem is a generalization of Theorem 14 in complex valued $b$-metric spaces.

Theorem 19. Let $(X, d)$ be a complete complex valued $b$-metric space with the coefficient $s \geq 1$ and let $S, T: X \rightarrow X$ be mappings satisfying

$$
d(S x, T y) \lesssim \frac{a[d(x, S x) d(x, T y)+d(y, T y) d(y, S x)]}{d(x, T y)+d(y, S x)},
$$

for all $x, y \in X$, where sa $\in[0,1)$. Then $S$, $T$ have a unique common fixed point in $X$.

Proof. For any arbitrary point, $x_{0} \in X$. Define sequence $\left\{x_{n}\right\}$ in $X$ such that

$$
x_{2 n+1}=S x_{2 n}, x_{2 n+2}=T x_{2 n+1}, \quad \text { for } k=0,1,2,3, \ldots
$$

Now, we show that the sequence $\left\{x_{n}\right\}$ is Cauchy. Let $x=x_{2 n}$ and $y=x_{2 n+1}$ in (33); we have

$$
\begin{aligned}
d\left(x_{2 n+1}, x_{2 n+2}\right)= & d\left(S x_{2 n}, T x_{2 n+1}\right) \\
\lesssim & a\left[d\left(x_{2 n}, S x_{2 n}\right) d\left(x_{2 n}, T x_{2 n+1}\right)\right. \\
& \quad+d\left(x_{2 n+1}, T x_{2 n+1}\right) d\left(x_{2 n+1}, S x_{2 n}\right] \\
\times & \left(d\left(x_{2 n}, T x_{2 n+1}\right)+d\left(x_{2 n+1}, S x_{2 n}\right)\right)^{-1} \\
= & a\left[d\left(x_{2 n}, x_{2 n+1}\right) d\left(x_{2 n}, x_{2 n+2}\right)\right. \\
& \quad+d\left(x_{2 n+1}, x_{2 n+2}\right) d\left(x_{2 n+1}, x_{2 n+1}\right] \\
\times & \left(d\left(x_{2 n}, x_{2 n+2}\right)+d\left(x_{2 n+1}, x_{2 n+1}\right)\right)^{-1},
\end{aligned}
$$

which implies that

$$
\begin{aligned}
& \left|d\left(x_{2 n+1}, x_{2 n+2}\right)\right| \\
& \leq a\left[\left|d\left(x_{2 n}, x_{2 n+1}\right)\right|\left|d\left(x_{2 n}, x_{2 n+2}\right)\right|\right. \\
& \left.+\left|d\left(x_{2 n+1}, x_{2 n+2}\right)\right|\left|d\left(x_{2 n+1}, x_{2 n+1}\right)\right|\right] \\
& \times\left(\left|d\left(x_{2 n}, x_{2 n+2}\right)+d\left(x_{2 n+1}, x_{2 n+1}\right)\right|\right)^{-1} \\
& =\frac{a\left|d\left(x_{2 n}, x_{2 n+1}\right)\right|\left|d\left(x_{2 n}, x_{2 n+2}\right)\right|}{\left|d\left(x_{2 n}, x_{2 n+2}\right)\right|} \\
& =a\left|d\left(x_{2 n}, x_{2 n+1}\right)\right| \text {, }
\end{aligned}
$$

and hence

$$
\left|d\left(x_{2 n+1}, x_{2 n+2}\right)\right| \leq a\left|d\left(x_{2 n}, x_{2 n+1}\right)\right| .
$$

Similarly, we can see that

$$
\left|d\left(x_{2 n+2}, x_{2 n+3}\right)\right| \leq a\left|d\left(x_{2 n+1}, x_{2 n+2}\right)\right| .
$$

Since $s a<1$ and $s \geq 1$, we get $a<1$.
Therefore, for all $n \geq 0$, consequently, we have

$$
\begin{aligned}
\left|d\left(x_{2 n+1}, x_{2 n+2}\right)\right| & \leq a\left|d\left(x_{2 n}, x_{2 n+1}\right)\right| \\
& \leq a^{2}\left|d\left(x_{2 n-1}, x_{2 n}\right)\right| \\
& \leq \cdots \leq a^{2 n+1}\left|d\left(x_{0}, x_{1}\right)\right| .
\end{aligned}
$$

Thus for any $m>n, m, n \in \mathbb{N}$, we have

$$
\begin{aligned}
&\left|d\left(x_{n}, x_{m}\right)\right| \\
& \leq s\left|d\left(x_{n}, x_{n+1}\right)\right|+s\left|d\left(x_{n+1}, x_{m}\right)\right| \\
& \leq s\left|d\left(x_{n}, x_{n+1}\right)\right|+s^{2}\left|d\left(x_{n+1}, x_{n+2}\right)\right|+s^{2}\left|d\left(x_{n+2}, x_{m}\right)\right| \\
& \leq s\left|d\left(x_{n}, x_{n+1}\right)\right|+s^{2}\left|d\left(x_{n+1}, x_{n+2}\right)\right| \\
&+s^{3}\left|d\left(x_{n+2}, x_{m}\right)\right|+s^{3}\left|d\left(x_{n+2}, x_{m}\right)\right| \\
& \leq s\left|d\left(x_{n}, x_{n+1}\right)\right|+s^{2}\left|d\left(x_{n+1}, x_{n+2}\right)\right|+s^{3}\left|d\left(x_{n+2}, x_{m}\right)\right| \\
&+\cdots+s^{m-n-2}\left|d\left(x_{m-3}, x_{m-2}\right)\right| \\
&+s^{m-n-1}\left|d\left(x_{m-2}, x_{m-1}\right)\right|+s^{m-n-1}\left|d\left(x_{m-1}, x_{m}\right)\right| \\
& \leq s\left|d\left(x_{n}, x_{n+1}\right)\right|+s^{2}\left|d\left(x_{n+1}, x_{n+2}\right)\right|+s^{3}\left|d\left(x_{n+2}, x_{m}\right)\right| \\
&+\cdots+s^{m-n-2}\left|d\left(x_{m-3}, x_{m-2}\right)\right| \\
&+s^{m-n-1}\left|d\left(x_{m-2}, x_{m-1}\right)\right|+s^{m-n}\left|d\left(x_{m-1}, x_{m}\right)\right| .
\end{aligned}
$$

By using (39), we get

$$
\begin{aligned}
& \left|d\left(x_{n}, x_{m}\right)\right| \\
& \leq \quad s a^{n}\left|d\left(x_{0}, x_{1}\right)\right|+s^{2} a^{n+1}\left|d\left(x_{0}, x_{1}\right)\right|+s^{3} a^{n+2}\left|d\left(x_{0}, x_{1}\right)\right| \\
& \quad+\cdots+s^{m-n-2} a^{m-3}\left|d\left(x_{0}, x_{1}\right)\right| \\
& \quad+s^{m-n-1} a^{m-2}\left|d\left(x_{0}, x_{1}\right)\right|+s^{m-n} a^{m-1}\left|d\left(x_{0}, x_{1}\right)\right| \\
& =\sum_{i=1}^{m-n} s^{i} a^{i+n-1}\left|d\left(x_{0}, x_{1}\right)\right| .
\end{aligned}
$$

Therefore,

$$
\begin{aligned}
\left|d\left(x_{n}, x_{m}\right)\right| & \leq \sum_{i=1}^{m-n} s^{i+n-1} a^{i+n-1} \mid d\left(x_{0},\right. \\
& =\sum_{t=n}^{m-1} s^{t} a^{t}\left|d\left(x_{0}, x_{1}\right)\right| \\
& \leq \sum_{t=n}^{\infty}(s a)^{t}\left|d\left(x_{0}, x_{1}\right)\right| \\
& =\frac{(s a)^{n}}{1-s a}\left|d\left(x_{0}, x_{1}\right)\right| .
\end{aligned}
$$

Now, since $s a<1$, we deduce

$$
\left|d\left(x_{n}, x_{m}\right)\right| \leq \frac{(s a)^{n}}{1-s a}\left|d\left(x_{0}, x_{1}\right)\right| \longrightarrow 0 \quad \text { as } m, n \rightarrow \infty .
$$

Thus, $\left\{x_{n}\right\}$ is a Cauchy sequence in $X$.

Since $X$ is complete, there exists some $u \in X$ such that $x_{n} \rightarrow u$ as $n \rightarrow \infty$. Assuming not, then there exist $z \in X$ such that

$$
|d(u, S u)|=|z|>0 .
$$


So by using the triangular inequality and (33), we get

$$
\begin{aligned}
& z=d(u, S u) \\
& \lesssim s d\left(u, x_{2 n+2}\right)+s d\left(x_{2 n+2}, S u\right) \\
& =s d\left(u, x_{2 n+2}\right)+s d\left(T x_{2 n+1}, S u\right) \\
& \lesssim s d\left(u, x_{2 n+2}\right)+\left(s a d(u, S u) d\left(u, T x_{2 n+1}\right)\right. \\
& \left.\quad+s a d\left(x_{2 n+1}, T x_{2 n+1}\right) d\left(x_{2 n+1}, S u\right)\right) \\
& \quad \times\left(d\left(u, T x_{2 n+1}\right)+d\left(x_{2 n+1}, S u\right)\right)^{-1} \\
& =s d\left(u, x_{2 n+2}\right)+\left(s a d(u, S u) d\left(u, x_{2 n+2}\right)\right. \\
& \left.\quad+s a d\left(x_{2 n+1}, x_{2 n+2}\right) d\left(x_{2 n+1}, S u\right)\right) \\
& \quad \times\left(d\left(u, x_{2 n+2}\right)+d\left(x_{2 n+1}, S u\right)\right)^{-1},
\end{aligned}
$$

which implies that

$$
\begin{aligned}
|z|=\mid & |d(u, S u)| \leq s\left|d\left(u, x_{2 n+2}\right)\right| \\
+ & \left(s a|d(u, S u)|\left|d\left(u, x_{2 n+2}\right)\right|\right. \\
& \left.+s a\left|d\left(x_{2 n+1}, x_{2 n+2}\right)\right|\left|d\left(x_{2 n+1}, S u\right)\right|\right) \\
& \times\left(\left|d\left(u, x_{2 n+2}\right)+d\left(x_{2 n+1}, S u\right)\right|\right)^{-1} .
\end{aligned}
$$

Taking the limit of (48) as $n \rightarrow \infty$, we obtain that $|z|=$ $|d(u, S u)| \leq 0$, a contradiction with (44). So $|z|=0$. Hence $S u=u$. Similarly, we obtain $T u=u$.

Now we show that $S$ and $T$ have unique common fixed point of $S$ and $T$. To show this, assume that $u^{*}$ is another common fixed point of $S$ and $T$. Then

$$
\begin{aligned}
d\left(u, u^{*}\right) & =d\left(S u, T u^{*}\right) \\
& \precsim \frac{a\left[d(u, S u) d\left(u, T u^{*}\right)+d\left(u^{*}, T u^{*}\right) d\left(u^{*}, S u\right)\right]}{d\left(u, T u^{*}\right)+d\left(u^{*}, S u\right)} \\
& \prec d\left(u, u^{*}\right) .
\end{aligned}
$$

This implies that $\left|d\left(x, x^{*}\right)\right| \leq 0$, and then $u=u^{*}$ which proves the uniqueness of common fixed point in $X$. This completes the proof.

Corollary 20. Let $(X, d)$ be a complete complex valued $b$ metric space with the coefficient $s \geq 1$ and let $T: X \rightarrow X$ be a mapping satisfying

$$
d(T x, T y) \lesssim \frac{a[d(x, T x) d(x, T y)+d(y, T y) d(y, T x)]}{d(x, T y)+d(y, T x)},
$$

for all $x, y \in X$, where $s \in[0,1)$. Then $T$ has a unique fixed point in $X$.

Corollary 21. Let $(X, d)$ be a complete complex valued $b$ metric space with the coefficient $s \geq 1$ and let $T: X \rightarrow X$ be a mapping satisfying

$$
\begin{aligned}
& d\left(T^{n} x, T^{n} y\right) \\
& \quad \lesssim \frac{a\left[d\left(x, T^{n} x\right) d\left(x, T^{n} y\right)+d\left(y, T^{n} y\right) d\left(y, T^{n} x\right)\right]}{d\left(x, T^{n} y\right)+d\left(y, T^{n} x\right)},
\end{aligned}
$$

for all $x, y \in X$, where sa $\in[0,1)$ and $n \in \mathbb{N}$. Then $T$ has a unique fixed point in $X$.

\section{Conflict of Interests}

The author declares that there is no conflict of interests regarding the publication of this paper.

\section{References}

[1] S. Banach, "Sur les operations dans les ensembles abstraits et leur application aux equations integrals," Fundamenta Mathematicae, vol. 3, pp. 133-181, 1922.

[2] I. A. Bakhtin, "The contraction principle in quasimetric spaces," Functional Analysis, vol. 30, pp. 26-37, 1989.

[3] K. Rao, P. Swamy, and J. Prasad, "A common fixed point theorem in complex valued $b$-metric spaces," Bulletin of Mathematics and Statistics Research, vol. 1, no. 1, 2013.

[4] A. Azam, B. Fisher, and M. Khan, "Common fixed point theorems in complex valued metric spaces," Numerical Functional Analysis and Optimization, vol. 32, no. 3, pp. 243-253, 2011.

[5] S. Bhatt, S. Chaukiyal, and R. C. Dimri, "Common fixed point of mappings satisfying rational inequality in complex valued metric space," International Journal of Pure and Applied Mathematics, vol. 73, no. 2, pp. 159-164, 2011.

[6] H. Aydi, M. Bota, E. Karapinar, and S. Mitrovic, "A fixed point theorem for set valued quasi- contractions in $b$-metric spaces," Fixed Point Theory and Its Applications, vol. 2012, article 88, 2012.

[7] J. Roshan, V. Parvaneh, S. Sedghi, N. Shobkolaei, and W. Shatanawi, "Common fixed points of almost generalized $(\psi, \varphi)_{s}$-contractive mappings in ordered $b$-metric spaces," Fixed Point Theory and Applications, vol. 2013, article 159, 2013.

[8] S. Datta and S. Ali, "A common fixed point theorem under contractive condition in complex valued metric spaces," International Journal of Advanced Scientific and Technical Research, vol. 6, no. 2, pp. 467-475, 2012.

[9] W. Sintunavarat and P. Kumam, "Generalized common fixed point theorems in complex valued metric spaces and applications," Journal of Inequalities and Applications, vol. 2012, article 84, 2012. 


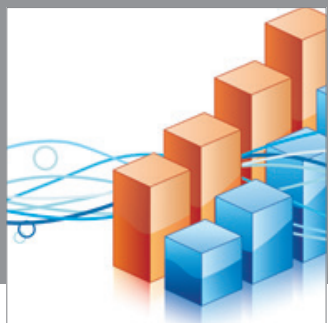

Advances in

Operations Research

mansans

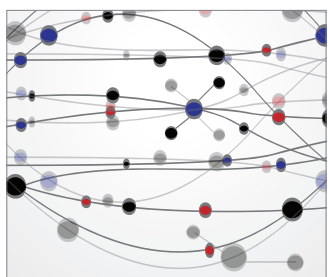

The Scientific World Journal
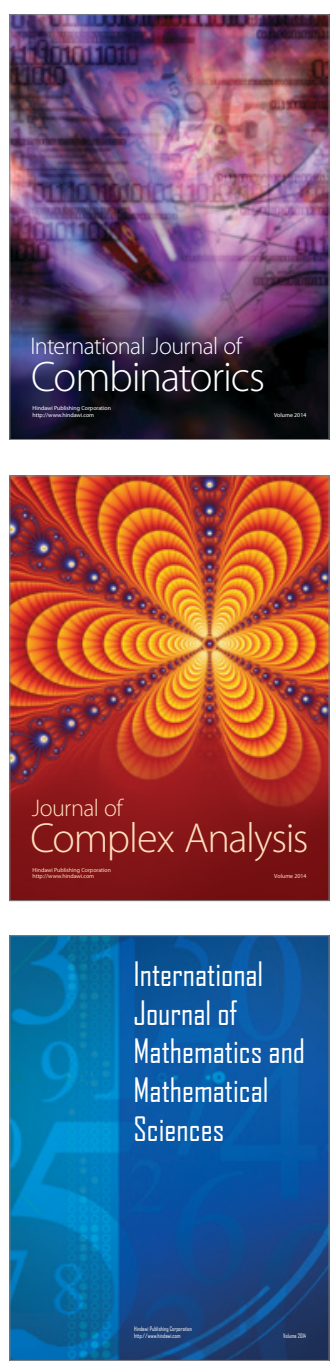
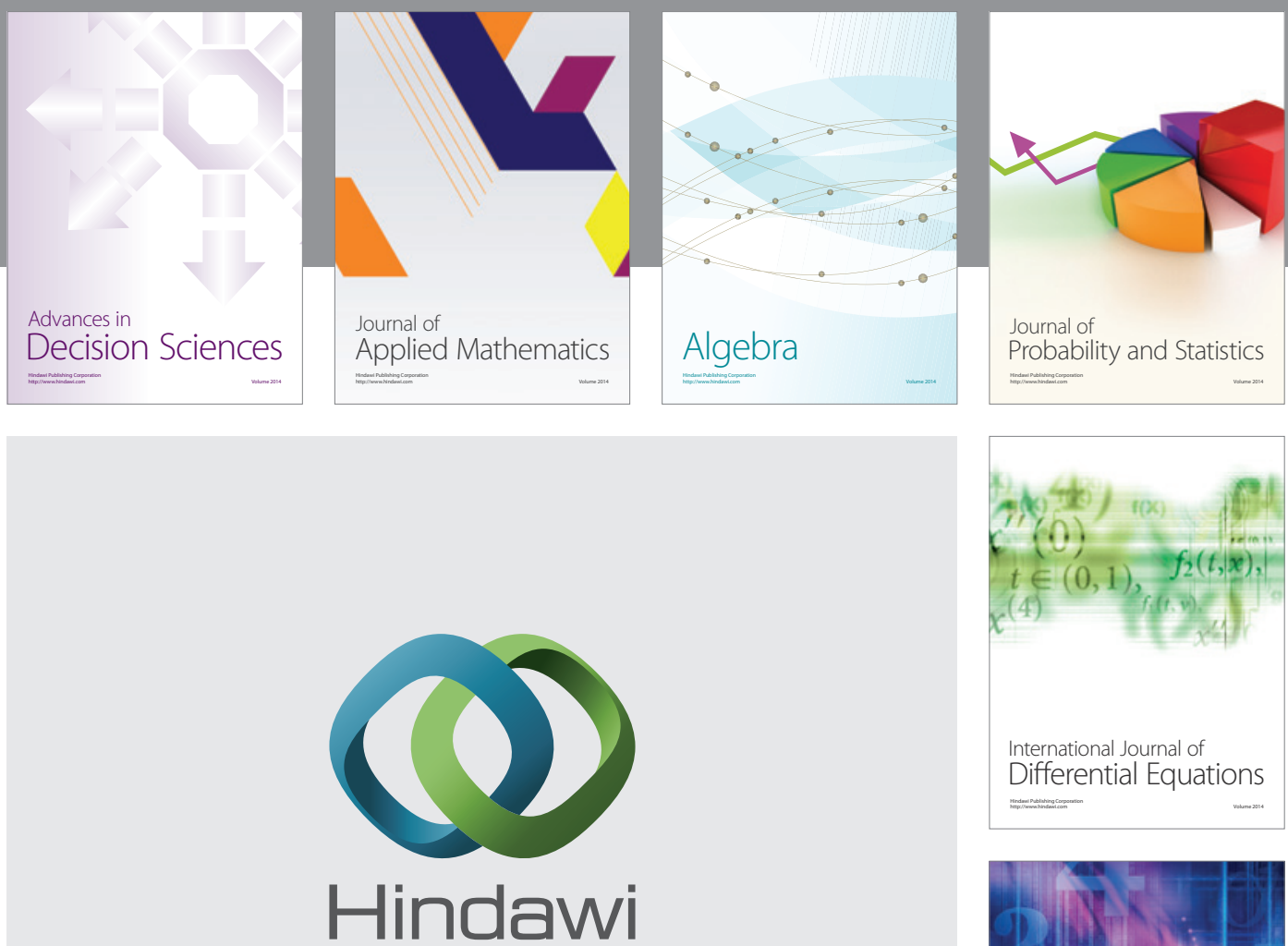

Submit your manuscripts at http://www.hindawi.com
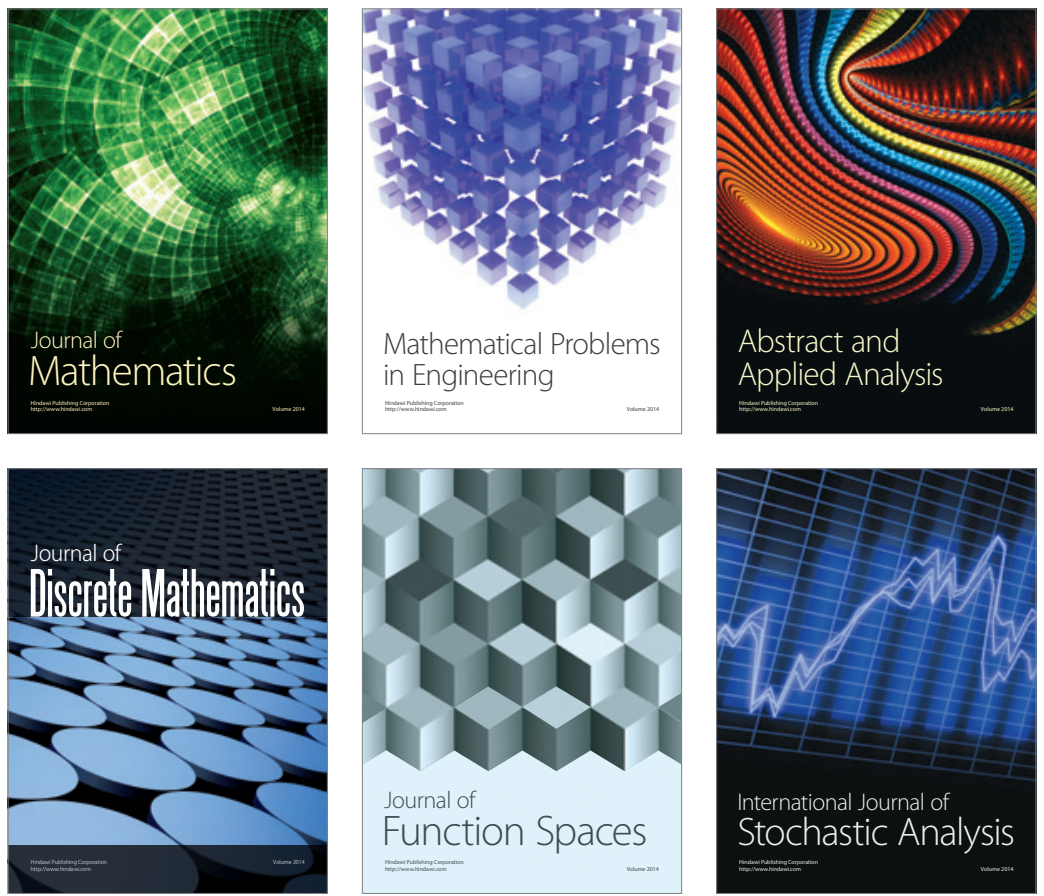

Journal of

Function Spaces

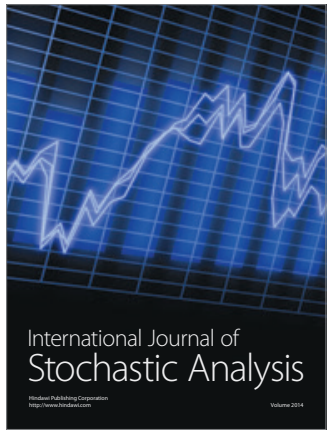

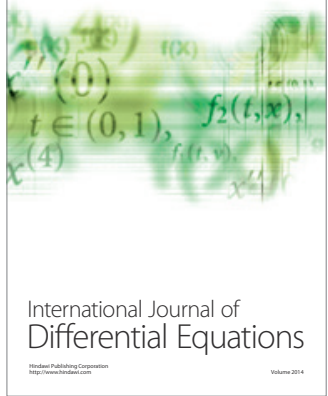
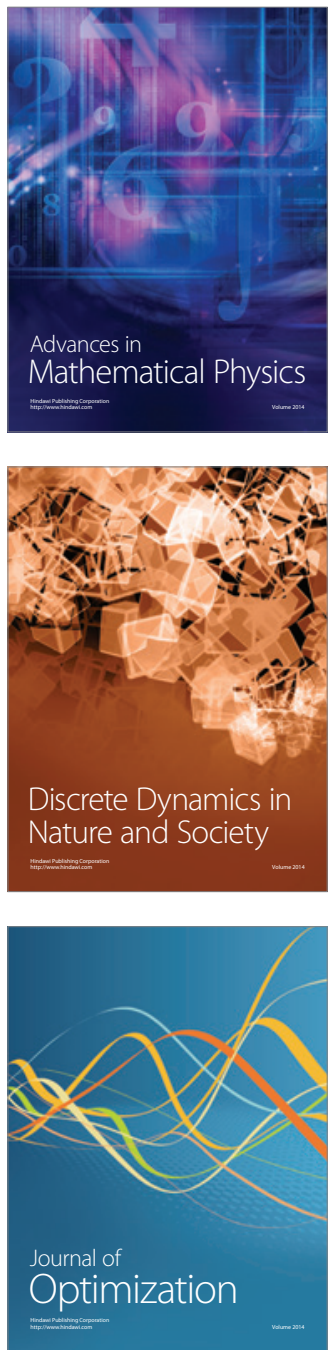